Editorial

\title{
Phenotypes of Klotho
}

Jyoti Misra Sen

Klotho gene was identified by Kuro-o et al. as hypomorphic mutant allele $[\mathrm{kl} / \mathrm{kl}]$ that decreased expression of Klotho protein leading to pre-mature aging phenotypes and dramatic shortening of lifespan [1]. By contrast, transgenic expression of Klotho enhanced lifespan [2]. Taken together with the observation that Klotho expression declines with normal aging in humans and mice [3], these studies revealed a compelling role for Klotho in aging.

$\mathrm{kl} / \mathrm{kl}$-driven aging phenotypes in $\mathrm{C} 3 \mathrm{H}, \mathrm{BALB} / \mathrm{c}$ and 129 genetic backgrounds correlated with altered mineral and vitamin $\mathrm{D}$ metabolic pathways, culminating in high levels of serum vitamin D [4]. Highest levels of vitamin $\mathrm{D}$ in serum, 5 to 6-fold higher compared to age matched controls, occur in 2-week old mutant mice, which then stabilize at approximately 3 -fold higher in mice that live past the next few weeks [4]. Much additional work has pointed to high levels of vitamin $\mathrm{D}$ as being critical for aging phenotypes in $\mathrm{kl} / \mathrm{kl}$ mice [5]. Thus, high vitamin D levels driven by low Klotho expression mediate aging-related phenotypes in $\mathrm{C} 3 \mathrm{H}, \mathrm{BALB} / \mathrm{c}$ and 129 mice. Surprisingly, transfer of the $\mathrm{kl} / \mathrm{kl}$ allele to a pure C57BL/6J background [B6-kl/kl] shows amelioration of aging phenotypes despite significantly reduced Klotho expression [6]. Concomitantly, the levels of vitamin D in the serum are also normalized in B6- $k l / k l$ mice [7], consistent with the notion that high vitamin $\mathrm{D}$ levels in the serum promote aging-related phenotypes when Klotho expression is reduced.

Vitamin D levels in the serum are controlled by balanced expression of two enzymes, Cyp24a1 and Cyp27b1, in the kidney. Furthermore, signaling mediated by Klotho and FGF-23 heterodimer binding to FGFR regulates expression of these two enzymes in the kidney [reviewed in 8]. These studies indicate that different levels of expression of Cyp24a1 and Cyp27b1could be responsible for maintenance of vitamin $\mathrm{D}$ levels in $\mathrm{C} 57 \mathrm{BL} / 6$ mice. To study the mechanism involved in regulation of vitamin $\mathrm{D}$ levels in the serum, expression of Cyp24al and Cyp27b1 was compared in mouse strains susceptible to aging-related phenotypes [C3H, BALB/c and 129] and one that does not show these phenotypes [C57BL/6]. Expression of Cyp27b1 was found to be comparable in all four strains. By contrast, basal expression of Cyp24al was found to be significantly higher in kidneys of $\mathrm{C} 57 \mathrm{BL} / 6$ mice compared to $\mathrm{C} 3 \mathrm{H}, \mathrm{BALB} / \mathrm{c}$ and 129 mouse strains [7].
Because signaling by Klotho via FGFR has been shown to regulate Cyp24a1, this observation suggests that low basal expression of Cyp24al in susceptible strains may sensitize them to aging-related phenotypes, by augmenting the importance of FGF-23 and Klotho dimerdependent Cyp24a1 expression to maintain healthy balance of vitamin D in the serum. Therefore, when Klotho is not expressed, the levels of vitamin D in the serum rise to dangerous levels leading to aging-related phenotypes and pre-mature death.

To address differential expression of Cyp24a1, chromatin structural features surrounding Cyp24al gene were compared in $\mathrm{C} 57 \mathrm{BL} / 6$ and susceptible $\mathrm{C} 3 \mathrm{H}, \mathrm{BALB} / \mathrm{c}$ and 129 strains. Remarkably, super-enhancer like regulatory regions showed genetic variations including deletions and epigenetic alterations that were associated with lower expression of Cyp24al in the kidney of $\mathrm{C} 3 \mathrm{H}$, $\mathrm{BALB} / \mathrm{c}$ and 129 mice compared to $\mathrm{C} 57 \mathrm{BL} / 6$ mice [7]. These observations suggest that genetic alterations in susceptible inbred strains, C3H, BALB/c and 129 result in lower basal expression of Cyp24a1, which renders the susceptible strains dependent on FGF-23/Klothomediated induced expression of Cyp24al to regulate serum vitamin D levels. Significantly higher vitamin D levels in the serum promote aging-related phenotypes in the absence of support from Klotho/FGF23-dependent induced expression of Cyp24al in susceptible genetic backgrounds [Figure 1].

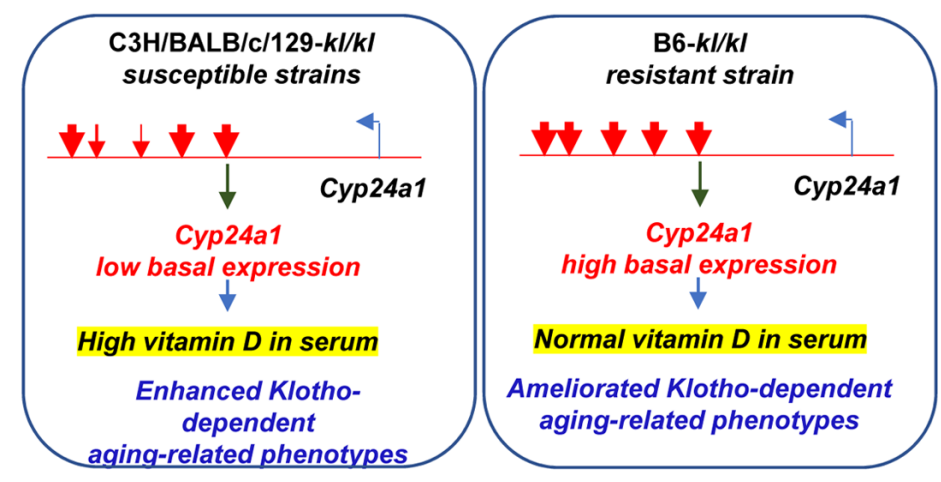

Figure 1. Genetic variations in the enhancer-like regions downstream of the Cyp24a1 gene regulate its basal expression in different strains of inbred mice. Deletions and substitutions in the putative Cyp24a1 regulatory sequences in susceptible $\mathrm{C} 3 \mathrm{H}, \mathrm{BALB} / \mathrm{c}$ and 129 mice lead to reduced basal expression of the gene, compared to resistant C57BL/6 mice, and render susceptible strains to high levels of vitamin $D$ in the serum and aging-related 
Specifically, this work provides a plausible mechanism by which genetic background of inbred mice influence the impact of Klotho on aging-related phenotypes. Because of genetic diversity, the functional impact of Klotho levels in aging phenotypes in humans may also be differentially affected by vitamin D levels. Studies of Singh et al. [7] suggest that dysregulation of vitamin D metabolism may cooperate with alterations in Klotho expression to impact aging.

\section{REFERENCES}

1. Kuro-o M, et al. Nature. 1997; 390:45-51. https://doi.org/10.1038/36285 PMID:9363890

2. Kurosu H, et al. Science. 2005; 309:1829-33. https://doi.org/10.1126/science.1112766 PMID:16123266

3. Arking $D E$, et al. Proc Natl Acad Sci USA. 2002; 99:856-61. https://doi.org/10.1073/pnas.022484299 PMID:11792841

4. Yoshida T, et al. Endocrinology. 2002; 143:683-89. https://doi.org/10.1210/endo.143.2.8657 PMID:11796525

5. Leibrock CB, et al. Sci Rep. 2016; 6:24879. https://doi.org/10.1038/srep24879 PMID:27109615

6. Phelps $M$, et al. Biogerontology. 2013; 14:729-39. https://doi.org/10.1007/s10522-013-9447-2 PMID:24030242

7. Singh A, et al. Aging Cell. 2019; 18:e12949. https://doi.org/10.1111/acel.12949 PMID:30920112

8. Takashi Y, Fukumoto S.. Trends Endocrinol Metab. 2018; 29:755-67. https://doi.org/10.1016/i.tem.2018.08.006 PMID:30217676

Jyoti Misra Sen: National Institute on Aging, National Institutes of Health, Baltimore, MD, 21224, USA

Correspondence: Jyoti Misra Sen

Email: jyoti-sen@nih.gov

Keywords: Klotho, Cyp24a1, vitamin D, epigenetics

Funding: This work was supported by Intramural research Program of the NIH, National Institute on Aging.

Copyright: Sen. This is an open-access article distributed under the terms of the Creative Commons Attribution License (CC BY 3.0), which permits unrestricted use, distribution, and reproduction in any medium, provided the original author and source are credited

Received: June 102019

Published: July 20, 2019 